\title{
A Novel Hybrid Range-free Approach to locate Sensor Nodes in 3D WSN using GWO-FA Algorithm
}

Prabhjot Singh

Chandigarh University

Nitin Mittal ( $\nabla$ mittal.nitin84@gmail.com )

Chandigarh University https://orcid.org/0000-0003-0758-2755

Parulpreet Singh

Lovely Professional University

\section{Research Article}

Keywords: Localization, WSNs, GWO-FA

Posted Date: July 26th, 2021

DOI: https://doi.org/10.21203/rs.3.rs-638905/v1

License: (1) This work is licensed under a Creative Commons Attribution 4.0 International License.

Read Full License 


\title{
A Novel Hybrid Range-free Approach to locate Sensor Nodes in 3D WSN using GWO- FA Algorithm
}

\author{
${ }^{1}$ Prabhjot Singh, ${ }^{1}$ Nitin Mittal ${ }^{*},{ }^{2}$ Parulpreet Singh \\ ${ }^{1}$ Deptt. of Electronics and Communication Engineering, Chandigarh University, Mohali- \\ 140413, Punjab, India \\ ${ }^{2}$ Deptt. of Electrical and Electronics Engineering, LPU, Phagwara
}

*Corresponding Author. Tel.: +91-9416987434

E-mail address: mittal.nitin84@gmail.com

\begin{abstract}
The precise node location of the sensor nodes is an essential requirement in Wireless Sensor Networks (WSNs) to determine the place or event occurring at a particular instant of time. In WSN, existing localization schemes considers two-dimensional (2D) space, while in actual life, sensor nodes are placed in three-dimensional (3D) space. In 3D localization, there are many research challenges, such as higher computational complexity, poor location prediction, lesser coverage, and depending only on fewer anchor nodes. To address various research issues in 3D environment we propose a range-free technique applied in an anisotropic scenario having Degree of Irregularity (DOI) as 0.01 using the concepts of fuzzy logic system (FLS). Anisotropic properties of nodes are considered to determine the efficiency of Grey wolf with Firefly algorithm. In our proposed scenario, the received signal strength (RSS) information is necessary among the target nodes and their corresponding anchor nodes for determining the location of target nodes using the information based on edge weights. These edge weights are further modelled using Hybrid GWO-FA to estimate the location of target nodes. The proposed algorithms is energy efficient as a single location aware node is used for localization. Further, the concept of virtual anchors is introduced that helps the algorithm to determine 3D positions.
\end{abstract}

Keywords- Localization, WSNs, GWO-FA.

\section{Introduction}

With the advancement in MEMS and wireless technologies have led to development of lowcost, lightweight, multi-functional and intelligent devices known as sensor nodes (SNs). Once deployed, these SNs are able to organize themselves to establish an ad hoc network, known as the wireless sensor network (WSN). WSNs can be used to track and detect physical phenomena in inaccessible and emergency situations, where it is almost impossible to deploy the sensors nodes. These SNs can detect the physical phenomena and, using multi-hop propagation, the sensed data is forwarded to a centralised device known as the base station or sink node. The sensing and distribution of sensed data requires the sensor, analogue to digital converter (ADC), processing unit, transceiver and battery/power supply to be equipped with these nodes. WSN implementations, however, require hundreds or thousands of SNs in the sensing field. In addition, because of their deployment in remote locations, the power source for such SNs cannot be replaced or energised. This includes optimum usage of SNs' computing and battery capacity so that WSN's overall lifespan can be increased.

In most of the WSN-applications, such as forest fire monitoring, surveillance, target tracking, large-terrains monitoring etc, requires an accurate knowledge of a particular location where the event has taken place. The sensed data is not useful in the absence of such knowledge. The location of sensed information can be either observed manually or deploying sensor nodes with in-built GPS [2-3] at known locations. Manual positioning of SNs cannot be made possible as 
they are used in an inaccessible region, while placing GPS or position finding on each node would increase the cost of using WSN and make it economically impossible to achieve [4]. Consequently, a wide variety of alternative approaches in literature [5-9] have been proposed in which random nodes (ANs) or beacon nodes are spread across the network in small quantities which are aware about their locations and location unaware nodes called target nodes determines their location with the help of anchor nodes. Localization approaches are classified broadly into two categories namely Range-based and Range-free. In range-based localization approach better accuracy at a cost of additional hardware integrated into SNs is achieved [10]. Few parameters such as noise and fading also degrades the performance of such technique. An alternative approach namely range-free technique is quite economical, because for location determination it only requires connectivity information. Due to their economic effectiveness, these localization techniques are quite common, but these techniques are less accurate as compared with range-based techniques. To improve the accuracy in range-free techniques is a big research challenge.

The literature reports the majority of location approaches are for two-dimensional (2D) localization [5-9], while the realistic scenario demands for SNs to be positioned in a threedimensional (3D) environment. Although identifying locations of SNs in three-dimensional environments is nevertheless more complex and difficult.

In this paper, we propose a hybrid GWO-FA optimization technique using Fuzzy Logic System (FLS) [11] for locating SNs in range-free 3D environment. In contrast to the other metaheuristics available in literature, the proposed algorithm provides improved performance in terms of coverage, and localization accuracy. Nodes are positioned in a random fashion in three -dimensional space and an anisotropic property is taken into the consideration. The RSS values between deployed nodes and an anchor node that works on the basis of distance measurements are modelled using FLS and further GWO-FA is used to reduce errors generated in a localization process.

The remaining portion of the article is categorised as: Section 2 provides the detailed explanation on 3D localization techniques. In Section 3, FLS, GWO-FA optimization used for localization to deal with range-free WSNs is presented. The Radio irregularity model (RIM) is presented in Section 4, with above mentioned algorithms. In Section 5, proposed algorithm GWO-FA with Fuzzy is presented. The results and comparative analysis is presented in Section 6 , and finally conclusion and future work is described in Section 7.

\section{Literature Review}

To solve the localization problems in WSNs, numerous localization algorithms are suggested. The connectivity information between an anchor and target node, and number of hop counts play a crucial role in range-free localization scheme for estimating the locations of unknown nodes. In range-free techniques, anchor node broadcast a beacon signal and the in-range target nodes sense that signal and maintain an account of the received signal strength. Further, simple centroid method is applied to determine the coordinates of target nodes. In [7], author proposed range-free method based on proximity information used in coarse-grained localization algorithm. In proposed algorithm, centroid method is used to calculate the 2D location of target node by taking the location of anchor node into consideration [12]. Other well-known existing range-free algorithms are Centroid (CL) [7], Semi-Definite Position (SDP) [8], Convex [9], Approximate Point-In-Triangulation (APIT) [10], DV-Hop [11], etc. Determining the 2D coordinates of target nodes using range-free schemes are widely available in literature. Whereas, obtaining an accurate 3D coordinates of target node is still an open research challenge. Main research contribution for 3D node localization using range-free methods in literature is as: In [13], authors proposed a hybrid localization algorithm by combining RSSI 
and hop distance information to calculate 3D coordinates of target node better accuracy. However, proposed scheme is not considered for an irregular network. In [14], two range free localization techniques based on RSS information are introduced with application of computational intelligence methods. FLS is used to model edge weights and further optimized using application of the genetic algorithm (GA) in first technique. Whereas, in second technique, application of neural networks (NN) is used to calculate the position by considering localization as single problem. In paper [15], authors extended a trifling localization strategy utilizing low cost and little area WSNs. This technique is completely dispersed and satisfies a high restriction authorization for the focus on sensor network. Authors in [16] offered a plan to improve the Monte-Carlo confinement strategy by orchestrating an adjusted hereditary calculation grounded on the amounts of the Least-Mean-Square (LMS). Authors in [17] extended a versatile iterative localization strategy dependent on the steepest gradient descent. In 3D WSNs [18], Authors introduced another strategy which is hybrid between the RSS and AoA localization methodology, where sensor nodes are arbitrarily scattered with unidentified transmission power and path loss exponents. In [19], authors proposed two 3D based rangefree localization schemes using HPSO and BBO in anisotropic environment of WSN and proposes that the non-linearity between RSS and distance can be reduced by FLS. In [20], two range-free 3D algorithms are proposed using application of bacterial foraging optimization (BFO) and invasive weed optimization (IWO). For better localization accuracy, edge weight between target node and their nearest anchor node are mapped using FLS, reduce computational complexity and non-linearity between RSS and distance. In [21], a range-free method is discussed for multi-hop transmission in anisotropic network. Hop count deviation in shortest path and direct path brings detored path between nodes. Path deviation is used to estimate the new distance for shortest path. Application of particle swarm optimization (PSO) is used for range-free 3D DV-HOP localization method is proposed. Proposed scheme improves the accuracy over the traditional DV-HOP method in [22]. A novel distance estimation scheme using centralized approach to reduce the computational and node density is proposed in [23]. To establish the 3D coordinates of target node, minimum four anchor nodes are required. It increases the computation complexity and anchor node density. RSS signal is affected due to the obstacles available in the environment. To overcome from the computational complexity and anchor node density, a concept of virtual anchor node is proposed in this paper. Once target node falls within range of anchor node, six virtual anchor nodes are projected in the proximity of this anchor node. Further, RSS and edge weight are mapped with FLS. Application of different evolutionary algorithms are incorporated to reduce the localization error.

The method proposed here have the following attributes:

1. RSS and edge weights of an anchor node are mapped using FLS to calculate the precise locations of the unknown node.

2. The concept of virtual anchor with their projection is done within proximity of anchor node.

3. A novel performance index based on proximity is proposed, to evaluate the proposed method.

\section{The Proposed Algorithm (GWOFA)}

\subsection{Grey wolf optimization}

Grey Wolf Algorithm (GWO) is swarm intelligence-based metaheuristic developed by Mirjalili et al. [24] based on Grey wolves hunting mechanism. All the group members follow a hierarchy which is divided on the bases of their power and position in the group and the division is as follow. The Alpha or the leader of the group is responsible for all the decisions making. The pack has to follow the decision of the alpha and they acknowledge and give 
respect to their Alpha by keeping their tails down. The Beta is the discipliner for the pack and also the advisor to the alpha. Beta is considered as the suitable substitute for the position of alpha in its absence. Beta also acts as a medium between the pack and the alpha, the decisions made by Alpha is passed on to the pack by the Beta and the feedback of the pack is again passed on to the Alpha by Beta only. The Delta wolves are the dominating wolves which are placed after Beta in the hierarchy table. These wolves dominate the Omega wolves that are lowest in the hierarchy.

The delta wolves are further divided into five groups. The Scouts are those wolves that are responsible for safety of the pack they act as soldiers who keep their watch on the boundaries of their territories and are always alert about their surroundings and the danger approaching. The Sentinels are those wolves whose duty is to protect the pack. These wolves are somewhere related to scout wolves. Then comes the hunter wolves as the name justifies the hunter wolves help the upper order wolves in hunting down the prey and helps in providing food to the pack. The Elder wolves are considered the most experienced ones who once use to be alpha of the pack or beta. They are all experienced in decision making, hunting techniques and a well aware about the pack. The last but not the least the caretaker wolves are the wolves that take care of the ill and the wounded, weak wolves of their pack. Now comes the last hierarchy of the pack that is the omega.

The omega wolves are the scapegoat of the pack that means these are the weakest link of the pack and are sometimes even blamed for the mis happenings in the pack. They have to follow the orders of the alpha and beta and they are even dominated by the delta wolves; the omega wolves are the lowest in the hierarchy in their position as well as power also. These wolves hunt in the following steps: Encircling, Searching and Attacking the prey.

During the encircling, the position of the grey wolf is modified around the prey within the search space, which can be written mathematically as

$$
\begin{aligned}
& D=|C . X p(t)-X(t)| \\
& X(t+1)=X_{p}(t)-A . D
\end{aligned}
$$

where $t$ indicates the present iteration, $X_{p}(t)$ and $X(t)$ are the location vectors of prey and grey wolf respectively. $A$ and $C$ are coefficient vectors determined as

$$
\begin{aligned}
& A=2 a \cdot r_{1}-a \\
& C=2 \cdot r_{2}
\end{aligned}
$$

where $r_{1}$ and $r_{2}$ are uniformly distributed random vectors. During hunting process, value of $a$ decreases linearly from 2 towards 0 and hence $A$ also decreases using equation (3).

The positions of omega wolves are changed with respect to the three fittest wolves of the pack as these have good knowledge regarding the location of prey. The equations used for hunting the prey are as follows:

$$
\begin{gathered}
D_{\alpha}=\left|C_{1} X_{\alpha}-X\right|, D_{\beta}=\left|C_{2} \cdot X_{\beta}-X\right|, D_{\delta}=\left|C_{3} \cdot X_{\delta}-X\right| \\
X_{1}=X_{\alpha}-A_{1} \cdot\left(D_{\alpha}\right), X_{2}=X_{\beta}-A_{2} \cdot\left(D_{\beta}\right), X_{3}=X_{\delta}-A_{3} \cdot\left(D_{\delta}\right) \\
X(t+1)=\frac{X_{1}(\mathrm{t})+X_{2}(\mathrm{t})+X_{3}(\mathrm{t})}{3}
\end{gathered}
$$

With the aim is to find the location of prey, wolves segregate themselves first and then unites together for attacking the prey and this condition is fulfilled by the parameter $A$ in GWO. The parameter $C$ helps and control the exploration rate of the searching agents and avoids stagnation by allocating a random. The basic pseudo code for GWO is presented in Algorithm 1.

\subsection{Firefly Algorithm (FA)}

Firefly Algorithm (FA) is based on concept of swarm intelligence, given by Yang [25]. FA is inspired by the light flashing behaviour of fireflies and main aim for fireflies flash is to work as a signal for other flies to get attracted to it. These population of fireflies show some 
characteristic activities such as luminary flashing, attracting the partner, communication and warning about the predicator. Xin She Yang assumed few points to formulate the algorithm:

1. Fireflies are Unisexual, which means one firefly gets attracted to other fireflies.

2. Brightness is related to the attractiveness, if we consider two fireflies then the one with least brightness will attract the one with more brightness.

3. As the distance increases, the brightness decreases.

4. If there are no fireflies left that are considered brighter than all the fireflies will move randomly in any direction.

5. Firefly's brightness is analysed by the fitness value of the fitness function.

This Firefly Algorithm follows some parameters such as: Attractiveness, Absorption, and Randomization

Attractiveness parameter explains the concept of light intensity between two flies and is defined using an exponential function given by:

$$
I(r)=I_{0} \exp \left(-\gamma \cdot r^{2}\right)
$$

where $I_{0}$ is the value of light intensity at $r=0 ; I$ is the light intensity at distance $r$ from a firefly; and $\gamma$ is the coefficient of light absorption. The absorption parameter changes the value of attractiveness from zero (0) to infinity.

As the attractiveness of fireflies is proportional to the light intensity observed by adjacent fireflies, the attractiveness can be represented as follows:

$$
\beta=\beta_{0} \exp \left(-\gamma \cdot r^{2}\right)
$$

where $\beta_{0}$ is the attractiveness constant at $r=0$. The Cartesian distance is the distance between fireflies $i$ and $j$ can be expressed as:

$$
r_{i j}=\left\|X_{i}-X_{j}\right\|
$$

The firefly's $i$ motion as drawn to another brighter firefly's $j$ can be depicted as follows:

$$
\Delta X_{i}=\beta_{0} e^{-\gamma \cdot r_{i j}^{2}}\left(X_{j}^{t}-X_{i}^{t}\right)+\alpha\left(N_{\text {rand }}-0.5\right)
$$

where $t$ is the iteration. The term $\alpha\left(N_{\text {rand }}-0.5\right)$ is the randomization and the randomization parameter is analysed using Gaussian distribution. The next move of i's Firefly can be updated as follows:

$$
X_{i}^{t+1}=X_{i}^{t}+\Delta X_{i}=X_{i}^{t}+\beta_{0} e^{-\gamma \cdot r_{i j}^{2}}\left(X_{j}^{t}-X_{i}^{t}\right)+\propto\left(N_{\text {rand }}-0.5\right)
$$

Talking about random walk, if the parameter $\beta_{0}$ is considered zero, then there is random walk (i.e. when the fireflies move towards the best solution with best cost in that iteration) which is corresponding to the randomization parameter.

The firefly algorithm deals highly non-linear problems naturally. The algorithm doesn't require a good solution to begin its iteration process. The speed of convergence in firefly algorithm is very high which means that the probability to reach the optimal solution is high in the case of firefly algorithm. However, the disadvantage of FA is that there is high chances of getting trapped in local optimal.

\subsection{Proposed Hybrid GWOFA}

The GWO algorithm's exploration and exploitation processes have been modified in this algorithm. Exploration and exploitation are the ones that determine the functionality of an algorithm. Any algorithm that has a good balance of both of these operations can locate the global solution quickly. As a result, the GWO must be strengthened in order to improve its exploitation.

The standard GWO operates on randomly generated original search agents and using parameters $A$ and $C$, grey wolves explore the area of interest and reinforce the exploration and exploitation capability of the algorithm. Also, GWO has a few parameters to change certain 
variables to fulfil its local and global search capabilities. Although, GWO has solved a great number of engineering problems, it was found that the algorithm has some problems. The key problem facing GWO is local optima stagnation.

With respect to GWO's exploration and exploitation capabilities, it has good exploration equations but lacks proper equations for exploitation. This has been accomplished in the current work by employing generational division. This concept of generational separation is new, and there hasn't been much research done on it. This aids in fully exploiting and exploring the search space. We also know that in the instance of GWO, the general equations are highly effective in the exploration phase but ineffective in the exploitation phase. Using the Firefly method, dividing iterations into two equal halves allows the algorithm to use new equations for the second half (when more exploitation is necessary). The basic GWO equations are used in the first half of the solution space. FA is extremely efficient in exploitation operations, hence equation change based on FA will undoubtedly produce efficient outcomes.

An exploitation activity inspired by the FA principles is introduced to enhance the exploitation aspect to solve the problems arising from the weak exploitation process. In the suggested algorithm process, the FA definition affects the GWO algorithm and is called the GWOFA. This change will help to resolve the current weaknesses of the basic GWO characteristics of slow and premature convergence.

As discussed, the number of iterations in the proposed GWOFA algorithm is divided into two half's. In first half, the regular GWO's general equation (7) is used, and then in the second half a new search equation is used. The current equation is replaced with FA position update equation as follows.

$$
X_{\text {new }}(t+1)=\frac{X_{1 \text { new }}(\mathrm{t})+X_{2 n e w}(\mathrm{t})+X_{3 n e w}(\mathrm{t})}{3}
$$

where $X_{1 \text { new }}(\mathrm{t}), X_{2 n e w}(\mathrm{t})$, and $X_{3 n e w}(\mathrm{t})$ are the position vectors of alpha, beta and delta grey wolves inspired from FA for second half of iterations.

$$
\begin{aligned}
& X_{1 \text { new }}=X_{1}+\beta_{0} e^{-\gamma \cdot r_{1 j}^{2}}\left(X_{j}-X_{1}\right)+\propto\left(N_{\text {rand }}-0.5\right) \\
& X_{\text {2new }}=X_{2}+\beta_{0} e^{-\gamma \cdot r_{2 j}^{2}}\left(X_{j}-X_{2}\right)+\propto\left(N_{\text {rand }}-0.5\right) \\
& X_{3 \text { new }}=X_{3}+\beta_{0} e^{-\gamma \cdot r_{3 j}^{2}}\left(X_{j}-X_{3}\right)+\propto\left(N_{\text {rand }}-0.5\right)
\end{aligned}
$$

Algorithm 2 shows a pseudo code of GWOFA algorithm.

\subsection{Fuzzy Logic System (FLS)}

It is a type of many-valued rationale, in which real estimations of variables might be any number in the vicinity of 0 and 1 . The term fuzzy logic was given in proposal of fuzzy set theory submitted by Lotfi Zadeh in 1965. This basically deals with an idea of halfway truth, where real value may lie between totally true and totally false. From control theory to artificial intelligence, fuzzy logic has been applied and is being applied by lot of researchers.

It enhances the robustness of the system. The fuzzy logic configuration of consists of input scaling factor, fuzzifier, inference engine, defuzzifier and output scaling factor, as shown in figure 1.

- Input/Output Scaling Factor:- Basically, it is defined as a nonlinear mapping of the input data set to the scaler output data. The input scaling factor in FLS is used for transformation between crisp input data and universe of discourses of the fuzzy input variables and output scaling factor is used to tune the output gainof the FLS. 
- Fuzzifier:- The gathered crisp input data set is fuzzified at this block. This conversion is done by using set of fuzzy linguistic variables, linguistic terms and membershipfunctions.

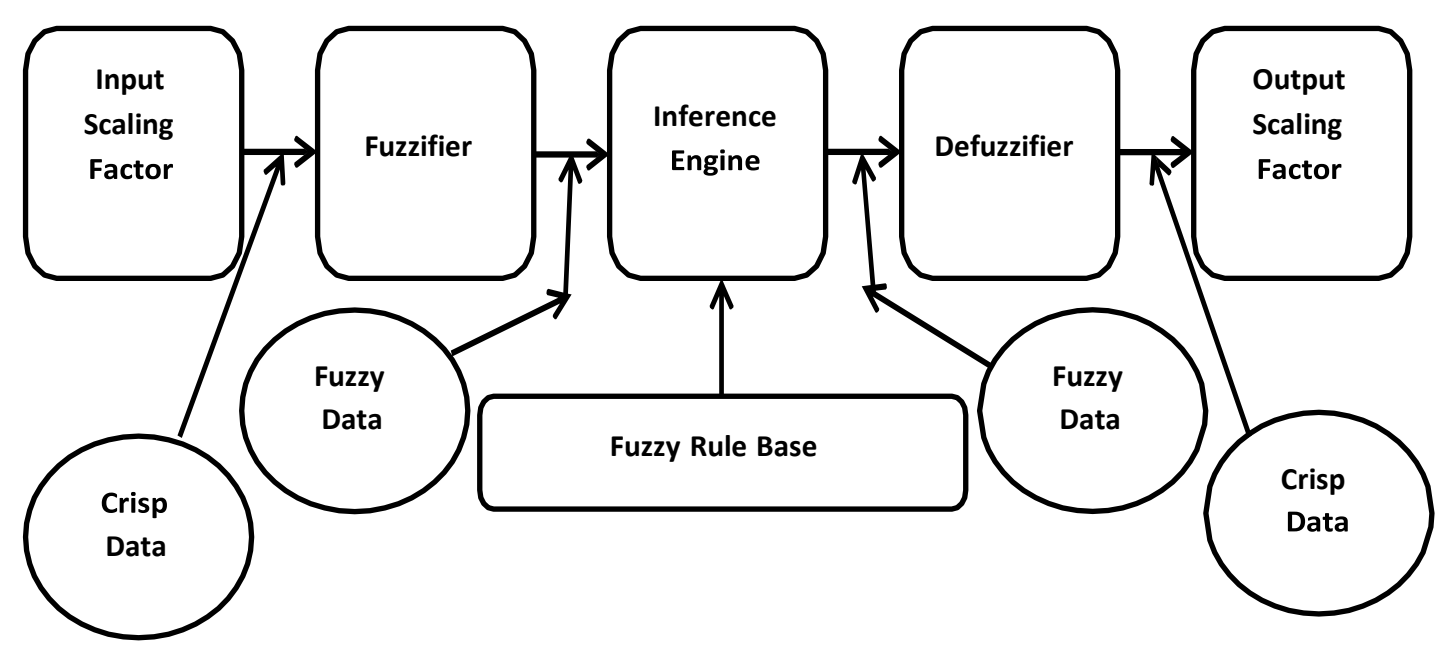

Figure 1: FLS

- Inference Engine:- The primary work of the inference engine is decision making.The output of the inference engine is always fuzzy irrespective of its input, i.e., whatever is the input (fuzzy or crisp) of the inference engine, the output is always fuzzy. Rule base, data base, decision making unit, fuzzification interface unit are thefunctional blocks of fuzzy inference system.

- Fuzzy Rules:- To control an output variable in this system a simple rule of if-then is used with a condition and a solution.

- Defuzzifier:- The defuzzification of the fuzzy data set is done at this block. This defuzzification is done by using membership functions and crisp output is taken from this block.

The fuzzy flow model is given in (5.17), where $\mathrm{x}$ represents an input variable that contains the RSS values from an anchor node and y signifies an output value, i.e. the edge weight for every target node is given by .

$$
R_{i}: \text { if } x \text { is } A_{i} \text { then } y \text { is } W_{i}
$$

Algorithm 1 Pseudo code for FLS

1: At initialization, define linguistic variables and terms

2: Membership function construction

3: Rule base construction

4: Fuzzification of crisp input data using membership functions

5: At inference system, evaluate the rule base and decision making

6: Combining the results of each rule base

7: Converting fuzzy data into crisp output using defuzzification

\section{Statistical Testing of GWOFA Algorithm}

Table 1 provides a set of ten CEC 2019 benchmark test functions known as the "100-Digit Challenge" [26] for GWOFA testing. All of these test functions can be scaled up or down. 
GWOFA's efficiency is assessed by comparing it to FPA, GWO, SSA, CS, DA, FDO, and SCA. With the support of 30 agents, each algorithm goes through 500 iterations. For each of the methods under consideration, the results are given in terms of the best, worst, mean, and standard deviation values for 30 independent runs. GWOFA outperforms other algorithms except in CEC03, CEC(06-07), and CEC(9-10) (see Table 2). Figure 2 shows the convergence graph and box plot of various approaches for various benchmark functions.

GWOFA's results for function CEC01 are better than other algorithms in terms of the best, worst, average, and median values achieved. GWO, CS, and GWOFA are all capable of identifying an optimal solution for function CEC02, but FDO is demonstrated to be the best. For the CEC03 function, most algorithms can give a nearly globally optimal solution, although FDO has been shown to be superior to other methods in terms of least standard deviation. GWOFA counteracts others in terms of fitness performance when it comes to CEC04 and CEC05. GWOFA's results for function CEC07 are the worst when compared to other methods. GWOFA's scores for function CEC08 are better in terms of best acquired fitness performance. GWOFA's results for the functions CEC09 and CEC10 are comparable to those of other approaches.

Figure 2 shows box plots of competing algorithms. These plots are used to evaluate efficiency of a algorithm in this scenario. In most cases, the suggested GWOFA is more cost-effective in terms of fitness values because the median value of GWOFA is lower. As a result, the overall performance of GWOFA has been found to be better than other optimization strategies.

Table 1 The 100-Digit Challenge Basic Test Functions [26]

\begin{tabular}{|c|l|c|c|c|}
\hline No. & \multicolumn{1}{|c|}{ Functions } & $\mathbf{F i}^{*}=\mathbf{F i}\left(\mathbf{x}^{*}\right)$ & $\mathbf{D}$ & Search Range \\
\hline 1 & $\begin{array}{l}\text { Storn's Chebyshev Polynomial Fitting } \\
\text { Problem }\end{array}$ & 1 & 9 & {$[-8192,8192]$} \\
\hline 2 & Inverse Hilbert Matrix Problem & 1 & 16 & {$[-16384,16384]$} \\
\hline 3 & Lennard-Jones Minimum Energy Cluster & 1 & 18 & {$[-4,4]$} \\
\hline 4 & Rastrigin's Function & 1 & 10 & {$[-100,100]$} \\
\hline 5 & Griewangk's Function & 1 & 10 & {$[-100,100]$} \\
\hline 6 & Weierstrass Function & 1 & 10 & {$[-100,100]$} \\
\hline 7 & Modified Schwefel's Function & 1 & 10 & {$[-100,100]$} \\
\hline 8 & Expanded Schaffer's F6 Function & 1 & 10 & {$[-100,100]$} \\
\hline 9 & Happy Cat Function & 1 & 10 & {$[-100,100]$} \\
\hline 10 & Ackley Function & & & {$[-100,100]$} \\
\hline
\end{tabular}

Table 2 CEC 2019 test suite for competitive algorithms

\begin{tabular}{llccccc}
\hline Function & Parameters & Best & Worst & Average & Median & Std. dev. \\
\hline & FPA & $1.37 \mathrm{E}+08$ & $1.20 \mathrm{E}+09$ & $5.03 \mathrm{E}+08$ & $4.41 \mathrm{E}+08$ & $2.78 \mathrm{E}+08$ \\
& GWO & $5.15 \mathrm{E}+04$ & $\mathbf{6 . 3 5 E}+\mathbf{0 8}$ & $1.14 \mathrm{E}+08$ & $3.46 \mathrm{E}+07$ & $1.64 \mathrm{E}+08$ \\
& SSA & $4.18 \mathrm{E}+08$ & $2.63 \mathrm{E}+10$ & $5.38 \mathrm{E}+09$ & $2.68 \mathrm{E}+09$ & $6.16 \mathrm{E}+09$ \\
\multirow{3}{*}{ CEC01 } & CS & $1.00 \mathrm{E}+10$ & $1.00 \mathrm{E}+10$ & $1.00 \mathrm{E}+10$ & $1.00 \mathrm{E}+10$ & $0.00 \mathrm{E}+00$ \\
& DA & $2.03 \mathrm{E}+09$ & $1.67 \mathrm{E}+11$ & $4.95 \mathrm{E}+10$ & $4.09 \mathrm{E}+10$ & $4.74 \mathrm{E}+10$ \\
& FDO & $7.32 \mathrm{E}+07$ & $7.66 \mathrm{E}+09$ & $1.34 \mathrm{E}+09$ & $6.37 \mathrm{E}+08$ & $1.72 \mathrm{E}+09$ \\
& SCA & $8.91 \mathrm{E}+05$ & $3.58 \mathrm{E}+10$ & $6.65 \mathrm{E}+09$ & $3.07 \mathrm{E}+09$ & $9.21 \mathrm{E}+09$ \\
& GWOFA & $\mathbf{3 . 8 6 E}+\mathbf{0 4}$ & $6.59 \mathrm{E}+08$ & $6.35 \mathrm{E}+07$ & $5.24 \mathrm{E}+06$ & $1.32 \mathrm{E}+08$ \\
\hline \multirow{2}{*}{ CEC02 } & FPA & $1.81 \mathrm{E}+01$ & $3.37 \mathrm{E}+01$ & $2.24 \mathrm{E}+01$ & $2.16 \mathrm{E}+01$ & $3.66 \mathrm{E}+00$ \\
& GWO & $1.73 \mathrm{E}+01$ & $1.73 \mathrm{E}+01$ & $1.73 \mathrm{E}+01$ & $1.73 \mathrm{E}+01$ & $2.69 \mathrm{E}-04$
\end{tabular}




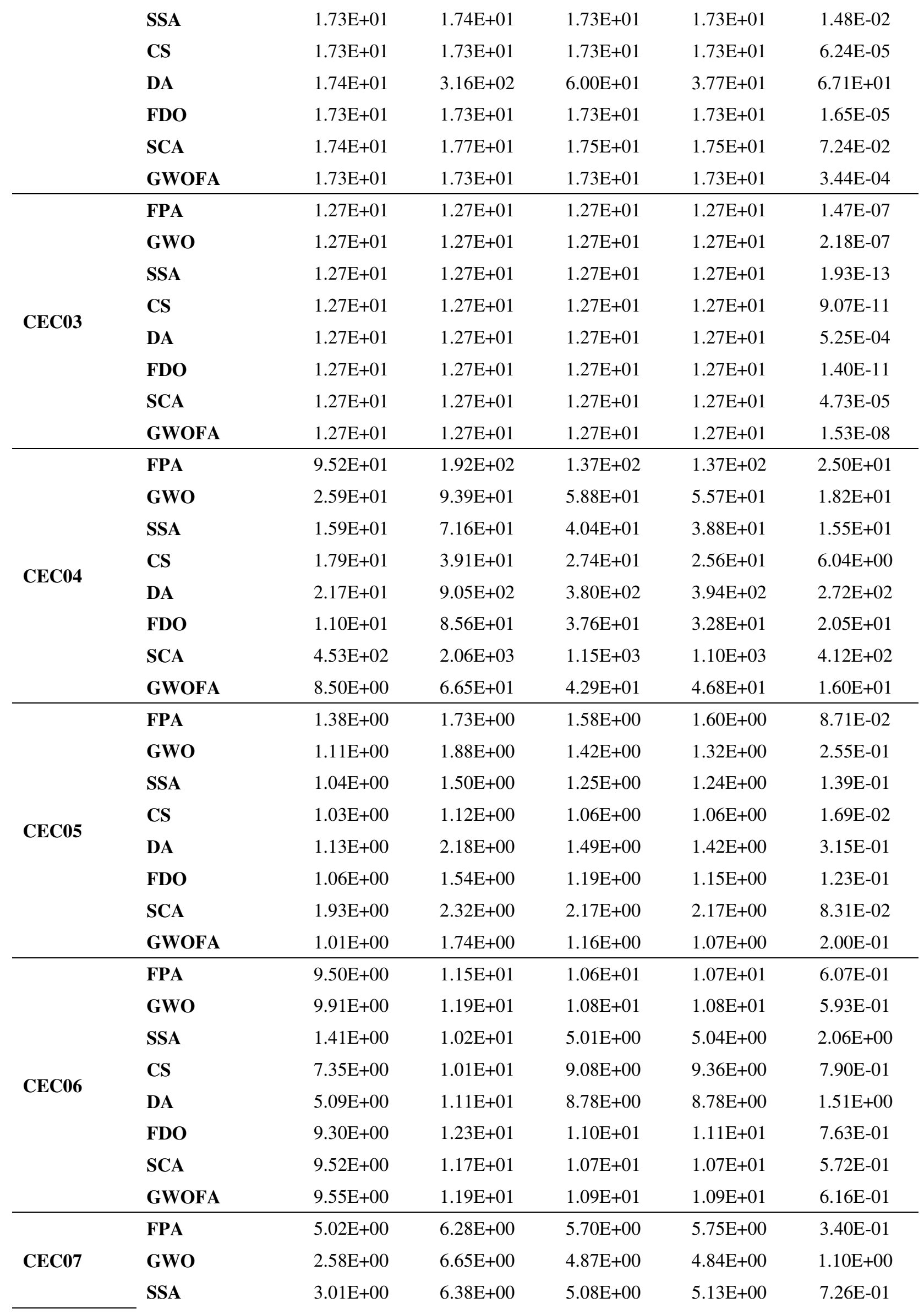




\begin{tabular}{|c|c|c|c|c|c|c|}
\hline & CS & $4.61 \mathrm{E}+00$ & $5.74 \mathrm{E}+00$ & $5.34 \mathrm{E}+00$ & $5.37 \mathrm{E}+00$ & $2.39 \mathrm{E}-01$ \\
\hline & DA & $5.12 \mathrm{E}+00$ & $6.78 \mathrm{E}+00$ & $5.84 \mathrm{E}+00$ & $5.92 \mathrm{E}+00$ & $4.21 \mathrm{E}-01$ \\
\hline & FDO & $3.36 \mathrm{E}+00$ & $6.27 \mathrm{E}+00$ & $5.05 \mathrm{E}+00$ & $5.12 \mathrm{E}+00$ & $6.27 \mathrm{E}-01$ \\
\hline & SCA & $4.88 \mathrm{E}+00$ & $6.91 \mathrm{E}+00$ & $5.85 \mathrm{E}+00$ & $5.91 \mathrm{E}+00$ & $5.42 \mathrm{E}-01$ \\
\hline & GWOFA & $6.87 \mathrm{E}+01$ & $1.01 \mathrm{E}+03$ & $5.10 \mathrm{E}+02$ & $5.39 \mathrm{E}+02$ & $2.78 \mathrm{E}+02$ \\
\hline \multirow{8}{*}{ CEC08 } & FPA & $5.11 \mathrm{E}+00$ & $6.48 \mathrm{E}+00$ & $5.77 \mathrm{E}+00$ & $5.77 \mathrm{E}+00$ & $3.41 \mathrm{E}-01$ \\
\hline & GWO & $3.45 \mathrm{E}+00$ & $6.75 \mathrm{E}+00$ & $5.18 \mathrm{E}+00$ & $5.27 \mathrm{E}+00$ & $9.31 \mathrm{E}-01$ \\
\hline & SSA & $4.05 \mathrm{E}+00$ & $6.14 \mathrm{E}+00$ & $5.34 \mathrm{E}+00$ & $5.37 \mathrm{E}+00$ & 4.66E-01 \\
\hline & CS & $4.82 \mathrm{E}+00$ & $5.66 \mathrm{E}+00$ & $5.26 \mathrm{E}+00$ & $5.33 \mathrm{E}+00$ & $1.94 \mathrm{E}-01$ \\
\hline & DA & $5.16 \mathrm{E}+00$ & $6.53 \mathrm{E}+00$ & $5.70 \mathrm{E}+00$ & $5.72 \mathrm{E}+00$ & $4.10 \mathrm{E}-01$ \\
\hline & FDO & $2.68 \mathrm{E}+00$ & $6.02 \mathrm{E}+00$ & $4.85 \mathrm{E}+00$ & $4.87 \mathrm{E}+00$ & $7.80 \mathrm{E}-01$ \\
\hline & SCA & $4.41 \mathrm{E}+00$ & $6.87 \mathrm{E}+00$ & $5.93 \mathrm{E}+00$ & $5.86 \mathrm{E}+00$ & $5.91 \mathrm{E}-01$ \\
\hline & GWOFA & $2.14 \mathrm{E}+00$ & $6.74 \mathrm{E}+00$ & $4.83 \mathrm{E}+00$ & $4.96 \mathrm{E}+00$ & $1.20 \mathrm{E}+00$ \\
\hline \multirow{8}{*}{ CEC09 } & FPA & $3.17 \mathrm{E}+00$ & $7.83 \mathrm{E}+00$ & $4.73 \mathrm{E}+00$ & $4.83 \mathrm{E}+00$ & $9.58 \mathrm{E}-01$ \\
\hline & GWO & $3.10 \mathrm{E}+00$ & $6.36 \mathrm{E}+00$ & $4.50 \mathrm{E}+00$ & $4.28 \mathrm{E}+00$ & $9.02 \mathrm{E}-01$ \\
\hline & SSA & $2.37 \mathrm{E}+00$ & $2.96 \mathrm{E}+00$ & $2.52 \mathrm{E}+00$ & $2.48 \mathrm{E}+00$ & $1.20 \mathrm{E}-01$ \\
\hline & CS & $2.49 \mathrm{E}+00$ & $2.95 \mathrm{E}+00$ & $2.74 \mathrm{E}+00$ & $2.75 \mathrm{E}+00$ & $1.34 \mathrm{E}-01$ \\
\hline & DA & $2.88 \mathrm{E}+00$ & $6.71 \mathrm{E}+00$ & $4.30 \mathrm{E}+00$ & $4.17 \mathrm{E}+00$ & $9.20 \mathrm{E}-01$ \\
\hline & FDO & $2.38 \mathrm{E}+00$ & $3.17 \mathrm{E}+00$ & $2.55 \mathrm{E}+00$ & $2.50 \mathrm{E}+00$ & $1.54 \mathrm{E}-01$ \\
\hline & SCA & $6.83 \mathrm{E}+00$ & $3.91 \mathrm{E}+02$ & $9.12 \mathrm{E}+01$ & $7.90 \mathrm{E}+01$ & $6.97 \mathrm{E}+01$ \\
\hline & GWOFA & $2.65 \mathrm{E}+00$ & $5.10 \mathrm{E}+00$ & $4.19 \mathrm{E}+00$ & $4.19 \mathrm{E}+00$ & $6.57 \mathrm{E}-01$ \\
\hline \multirow{8}{*}{ CEC10 } & FPA & $2.02 \mathrm{E}+01$ & $2.06 \mathrm{E}+01$ & $2.04 \mathrm{E}+01$ & $2.04 \mathrm{E}+01$ & $9.37 \mathrm{E}-02$ \\
\hline & GWO & $6.35 \mathrm{E}+00$ & $2.06 \mathrm{E}+01$ & $2.00 \mathrm{E}+01$ & $2.05 \mathrm{E}+01$ & $2.58 \mathrm{E}+00$ \\
\hline & SSA & $2.00 \mathrm{E}+01$ & $2.05 \mathrm{E}+01$ & $2.00 \mathrm{E}+01$ & $2.00 \mathrm{E}+01$ & 1.05E-01 \\
\hline & CS & $2.02 \mathrm{E}+01$ & $2.04 \mathrm{E}+01$ & $2.03 \mathrm{E}+01$ & $2.03 \mathrm{E}+01$ & 6.07E-02 \\
\hline & DA & $2.00 \mathrm{E}+01$ & $2.05 \mathrm{E}+01$ & $2.02 \mathrm{E}+01$ & $2.02 \mathrm{E}+01$ & $1.51 \mathrm{E}-01$ \\
\hline & FDO & $2.34 \mathrm{E}-08$ & $2.00 \mathrm{E}+01$ & $1.93 \mathrm{E}+01$ & $2.00 \mathrm{E}+01$ & $3.65 \mathrm{E}+00$ \\
\hline & SCA & $1.97 \mathrm{E}+01$ & $2.06 \mathrm{E}+01$ & $2.04 \mathrm{E}+01$ & $2.05 \mathrm{E}+01$ & $1.55 \mathrm{E}-01$ \\
\hline & GWOFA & $2.02 \mathrm{E}+01$ & $2.06 \mathrm{E}+01$ & $2.05 E+01$ & $2.05 E+01$ & $9.62 \mathrm{E}-02$ \\
\hline
\end{tabular}

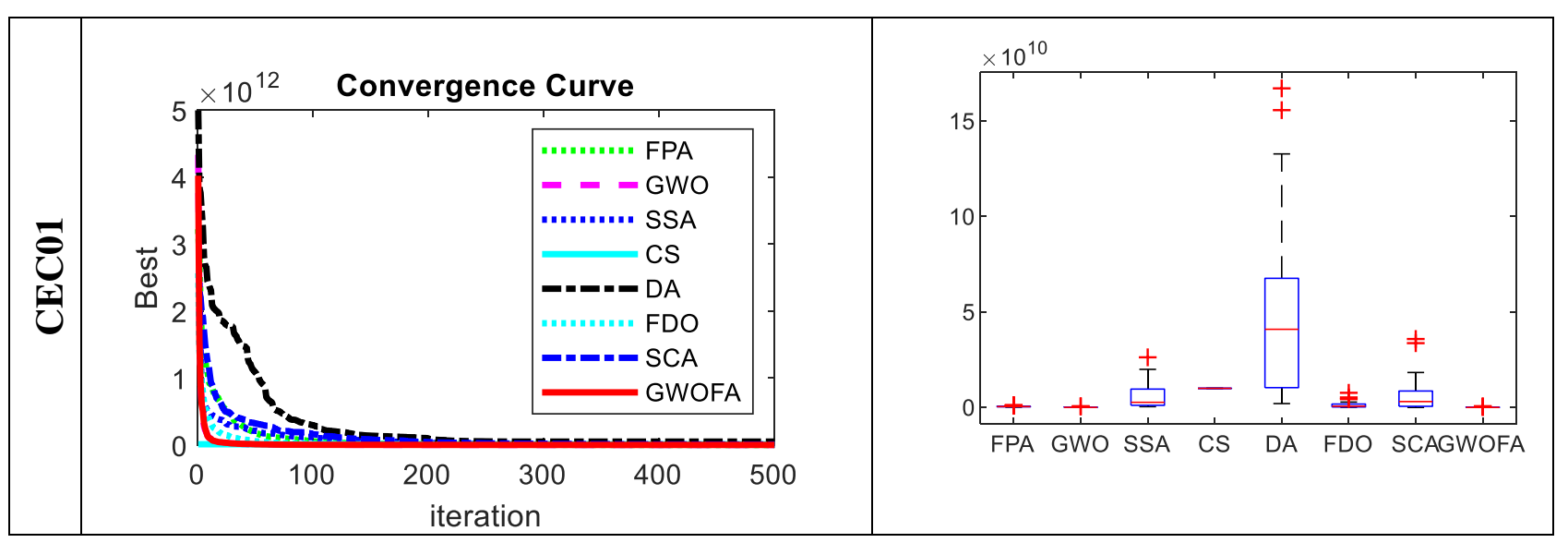




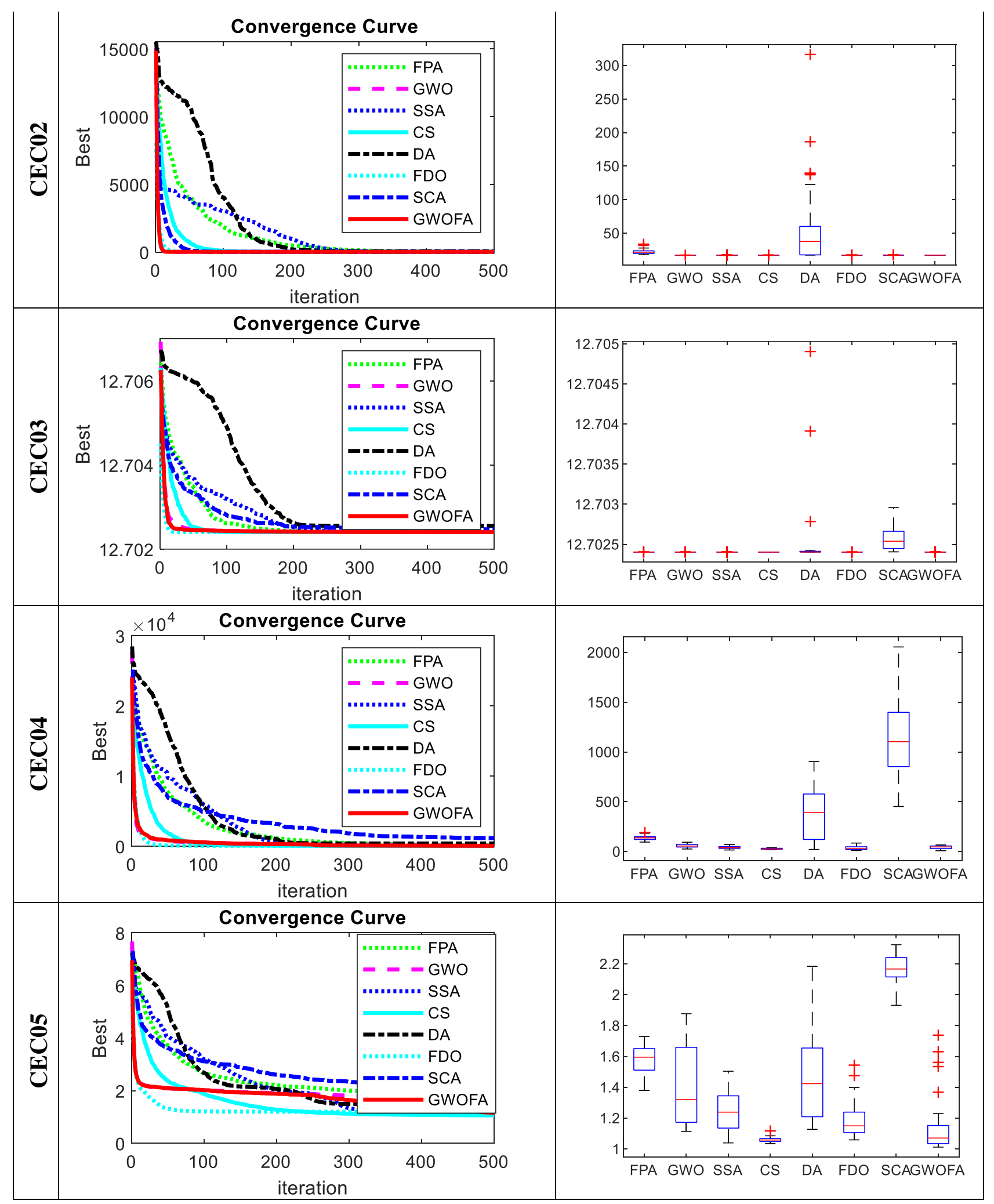




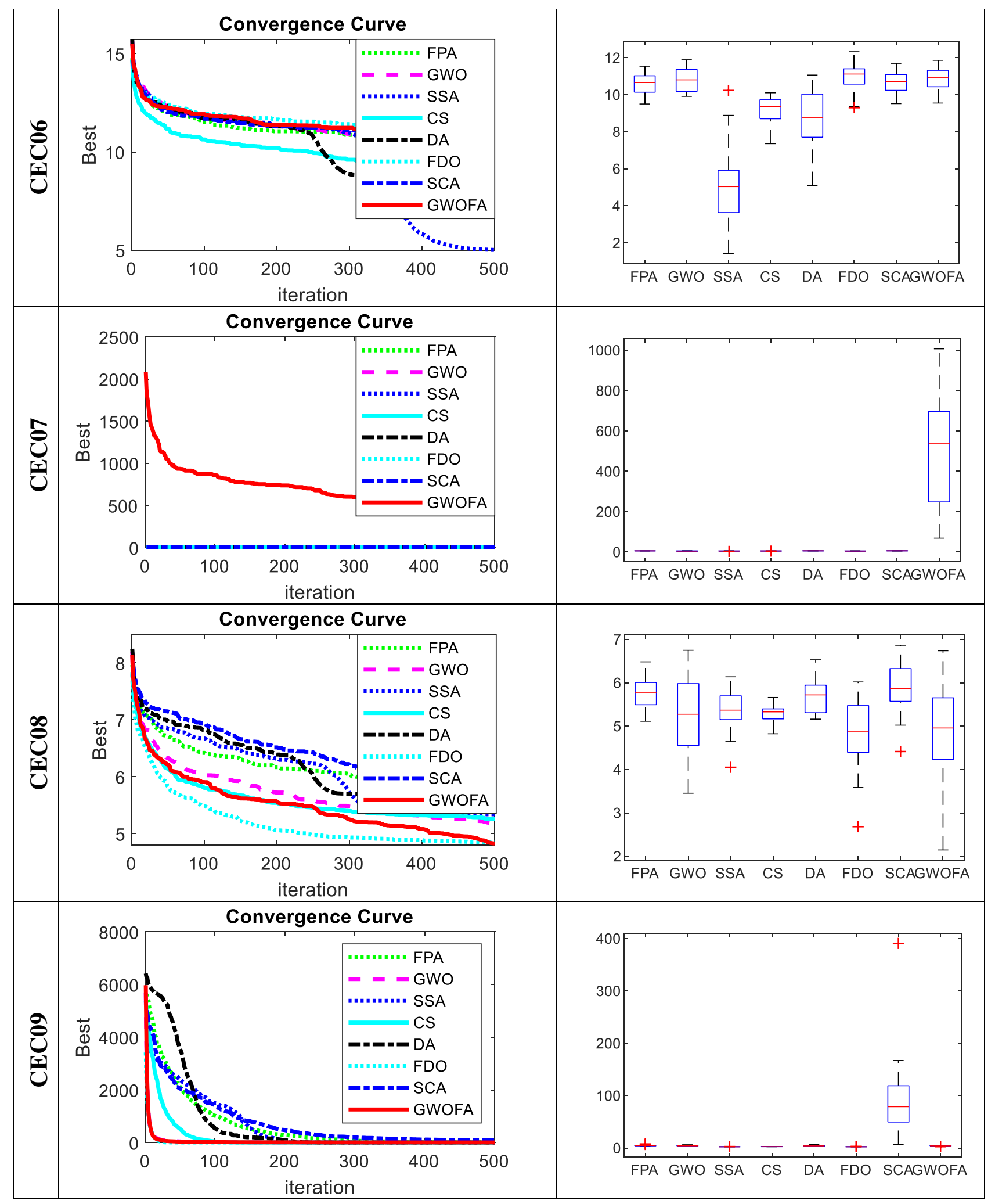




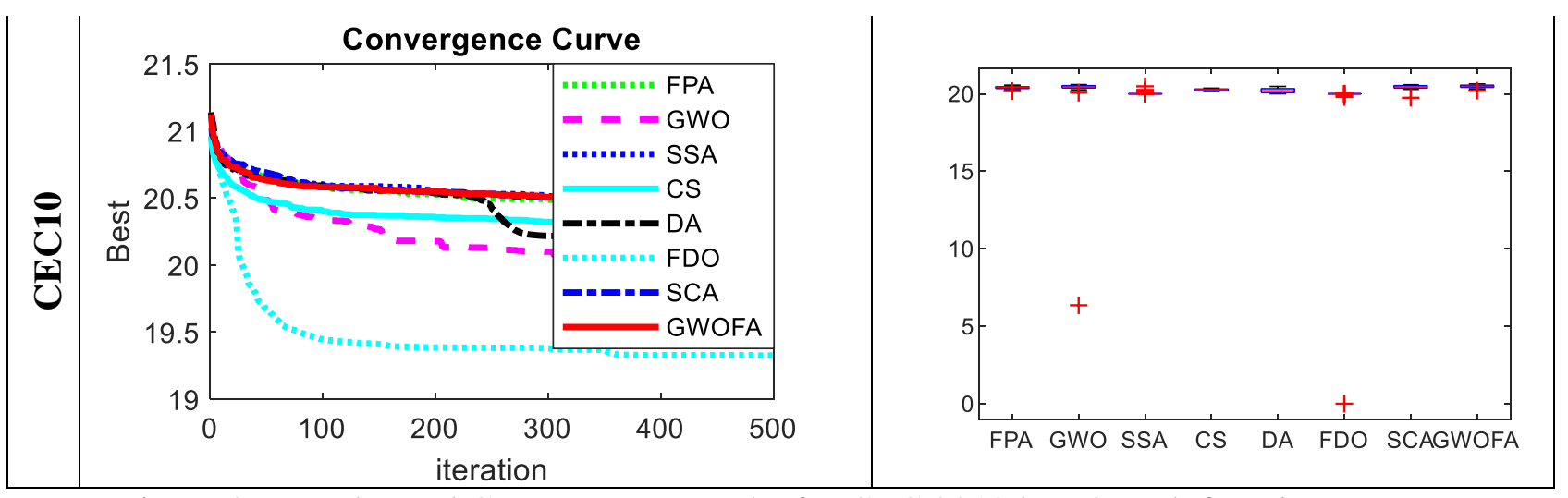

Figure 2: Boxplot and Convergence graphs for CEC 2019 benchmark functions

\section{Radio Irregularity Model}

Many existing localization strategies uses an ideal spectrum without considering DOI's. But it's not possible to achieve in practice. So radio-irregularity is one of the major aspect for studying a certain pattern in real situations. Radio irregularity is one of the primary concern that cannot be neglected in the wireless networks [29]. The effect of certain radio irregularity is also examined in our algorithm. As the variations in transmitting the signal with different RF powers and different path loss will lead to an irregularity in the radio pattern. To establish the anis-tropical properties of the propagation media the Radio Irregularity Model (RIM) is considered [30]. A DOI (degree of irregularity) parameter calculate the irregularity in radiation pattern.

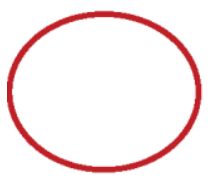

$\mathrm{DOI}=0$

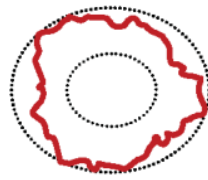

$\mathrm{DOI}=0.05$

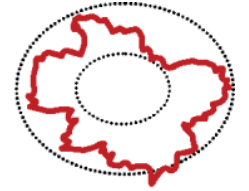

$\mathrm{DOI}=0.2$

FIGURE 3: Different values of DOI for Irregular radio patterns

\subsection{Problem Formulation}

A three layer structure is considered for locating sensor nodes in 3D scenario using anchor and target nodes positioned in a three layer structure. The anchor node is mobile and it moves randomly at regular intervals of time. The whole region is grouped into grids and under the range of an anchor node. The movement of an anchor node is assumed to cover the entire region. An anchor node transmits a beacon information and all unknown nodes receive it and transform it into the distance information. A range-free localization scheme is applied in this paper and the gathered RSSI information is sufficient enough to calculate distance.

As soon as the target node falls within the range of anchor node, a novel concept of selection of virtually deployed anchors is introduced (for each target node) to estimate their location. In every movement, an anchor node estimates an Euclidean distance. If Euclidean distance is less than anchor node's range then each target node is considered as localizable and possibly within a radius circle. Along with anchor node and six virtually assumed anchors are transmitted using same transmitting range (as minimum 3/4 nodes are neccessary to locate target nodes in 2D/3D environment. In range-free method only RSS information is sufficient enough to measure the distance between a target node and an anchor node. While gathering RSS information a signal may change its path due to blockage in environmental conditions. The mostly used propagation medium in WSN is path loss, lognormal, and Rayleigh fading. As RSS signal varies over 
shorter distances. All environmental parameters that degrades the performance are considered in our work. The received signal power is modelled using (17)

$$
P_{\text {rev } i}=\gamma^{2}\left(c \frac{P_{t x}}{d_{i j}{ }^{\alpha k_{i}}}\right) 10^{\frac{X_{s}}{10}}
$$

Where $\gamma$ represents Rayleigh distribution and accounts for multipath fading, $P_{t x}$ is transmitted power, $\mathrm{c}$ is determined by antenna characteristics, $\alpha$ represents path loss component, $10^{\frac{X_{S}}{10}}$ accounts for shadowing effect, $X_{S} \sim N\left(0, \sigma_{d}^{2}\right), k_{i}$ is path loss component due to anisotropic environment, and $\mathrm{N}$ represents a Gaussian random variable with zero men and variance $\sigma_{d}^{2}$. The measurement on the basis of RSS distance between the target and an anchor node is given by (18)

$$
d_{i j}=d \gamma^{\frac{2}{\alpha k_{i}}} e^{\frac{X_{s} \ln 10}{10 \alpha k_{i}}}
$$

\subsection{GWO-FA and Fuzzy Logic based Localization concept}

In our work a single anchor node is considered to locate all target nodes deployed in 3dimensional sensing field. The target nodes are dispersed in two different layers (i.e. bottom and middle layer) and an anchor is positioned at the top layer. The main task of using an anchor node is to transmit a beacon signal which help the target nodes to estimate their location. Whenever a target node drops within the range of an anchor node, it listens a beacon for a fixed time frame and collects RSS information of an anchor node. The Euclidean distance between an anchor and target node is calculated and the concept of virtual anchors is introduced in six distinct directions with an angle difference of $60^{\circ}$ each at an equal distance with respect to anchor node. Due to heterogeneity property radio propagation is spherical due to irregularity in radiation pattern and the transmission range is not equivalent in all directions. The anchor node's location is known either by GPS or any other means.

The below steps are followed for 3D location determination of a target node

1. The unknown sensor nodes are placed in between and beneath layers of a 3D cubic structure in a random fashion and a known sensor is placed on the top. The procedure of virtual anchors having parasol projection is considered in figure 4.

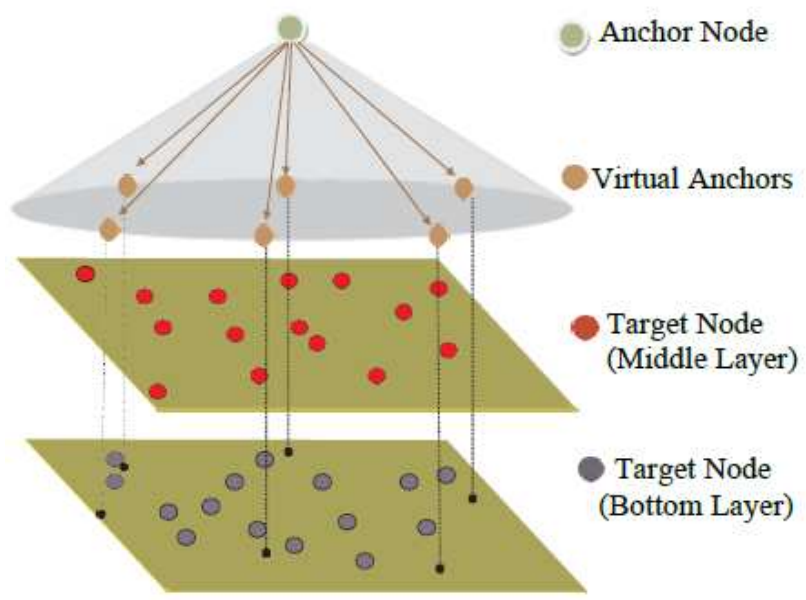

Figure 4: Parasol projection to localize moving target nodes 
2. Using $d_{i}^{\wedge}=d_{i}-\left(D_{p}+F\right)$, the distance among anchor and corresponding target node is calculated. Where, $d_{i}$ is the actual distance, $D_{p}$ is DOI adjusted path loss and F represents fading effect.

3. The RSS measurement is done using (19)

$$
R S S_{i j}=\frac{v}{d_{i j}^{\alpha}}
$$

4. Check whether or not the anchor node and virtual anchors are $\geq 4$

5. The edge weights are calculated and modeled using FLS.

6. To achieve accurate target node locations these edge weights and fuzzy sets are generated using various meta-heuristic algorithms.

7. The target node locations using the prior knowledge of edge weights is calculated using

$$
\left(x_{t}, y_{t}, z_{t}\right)=\frac{\left(w_{1} x_{1}\right)+\cdots+\left(w_{k} x_{k}\right)}{\sum_{i=1}^{k} w_{i}}, \frac{\left(w_{1} y_{1}\right)+\cdots+\left(w_{k} y_{k}\right)}{\sum_{i=1}^{k} w_{i}}, \frac{\left(w_{1} z_{1}\right)+\cdots+\left(w_{k} z_{k}\right)}{\sum_{i=1}^{k} w_{i}}
$$

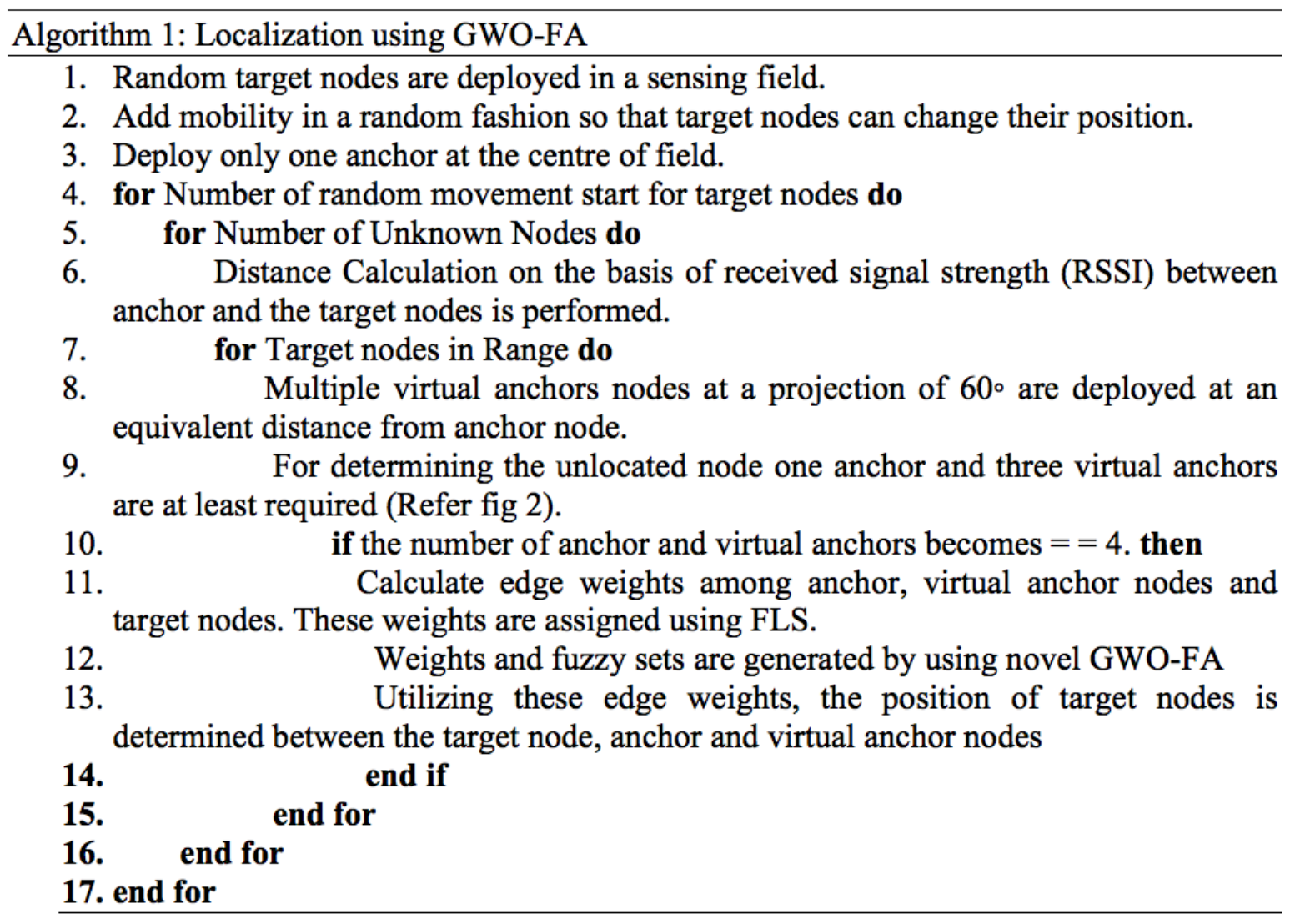

\subsection{Fuzzy Modeling for Edge weights with different meta-heuristics:}

In order to overcome the nonlinearity between distance and RSS measurements, the FLS with Mamdani implication is used to counteract the nonlinearity and develop a relation among RSS and edge weight of an anchor node. The algorithm 2 describing the flow of fuzzy modelling is given as:

Algorithm 2: Localization using Parasol Projection

1. Form of fuzzy model is given by (17) 
2. Value of input is $\left[0, R S S_{\max }\right]$, where $R S S_{\max }=100 \mathrm{db}$ represents the maximum value

3. This value is grouped into five membership functions

4. Value of output variable is taken as $\left[0, w_{\max }\right]$, where $w_{\max }=1$ represents the maxium edge weight

5. Membership functions of output variable generated by GWO-FA

6. Complete rule set have been made

7. To each rule set a degree of importance is applied

By using Mamdani implication operator, from these fuzzified values a single crisp set is taken

8. For FLS tuning, the output variable membership functions are optimized

The fuzzy flow model is given in (21), where $\mathrm{x}$ represents an input variable that contains the RSS values from a anchor node and y signifies an output value, i.e. for every target node the edge weight of an anchor node

$$
R_{i}: \text { if } x \text { is } A_{i} \text { then } y \text { is } W_{i}
$$

The input containing RSS values is grouped among five different membership functions using triangular, $\mathrm{S}$ type, and $\mathrm{Z}$ type function represented in fig.4. To complete the rule set the membership functions are categorised as $W_{i}$ is either very low, low, medium, high and either very high as given in Table 3 . The degree of importance for each and every rule to be applied is given by

$$
\text { Degree of Importance }=\mu(x) \times \mu(y)(22)
$$

\begin{tabular}{|c|c|c|}
\hline S. No. & Antecedent & Consequent \\
\hline 1 & When value of RSS is Very Low & Then Very Low EW \\
\hline 2 & When value of RSS is Very Low & Then Low EW \\
\hline 3 & When value of RSS is Very Low & Then Medium EW \\
\hline 4 & When value of RSS is Very Low & Then High EW \\
\hline 5 & When value of RSS is Very Low & Then Very High EW \\
\hline 6 & When value of RSS is Low & Then Very Low EW \\
\hline 7 & When value of RSS is Low & Then Low EW \\
\hline 8 & When value of RSS is Low & Then Medium EW \\
\hline 9 & When value of RSS is Low & Then High EW \\
\hline 10 & When value of RSS is Low & Then Very High EW \\
\hline 11 & When value of RSS is Medium & Then Very Low EW \\
\hline 12 & When value of RSS is Medium & Then Low EW \\
\hline 13 & When value of RSS is Medium & Then Medium EW \\
\hline 14 & When value of RSS is Medium & Then High EW \\
\hline 15 & When value of RSS is Medium & Then Very High EW \\
\hline 16 & When value of RSS is $\mathrm{High}$ & Then Very Low EW \\
\hline 17 & When value of RSS is $\mathrm{High}$ & Then Low EW \\
\hline 18 & When value of RSS is $\mathrm{High}$ & Then Medium EW \\
\hline 19 & When value of RSS is $\mathrm{High}$ & Then High EW \\
\hline 20 & When value of RSS is $\mathrm{High}$ & Then Very High EW \\
\hline 21 & When value of RSS is Very High & Then Very Low EW \\
\hline 22 & When value of RSS is Very High & Then Low EW \\
\hline 23 & When value of RSS is Very High & Then Medium EW \\
\hline 24 & When value of RSS is Very High & Then High EW \\
\hline 25 & When value of RSS is Very High & Then Very High EW \\
\hline
\end{tabular}

Table 3: Rule Base 
Table 4: Edge Weights (With Redundancy Removed)

\begin{tabular}{|c|c|c|}
\hline S.No. & Antecedent & Consequent \\
\hline 1 & When value of RSS is Very Low & Then Very Low EW \\
\hline 2 & When value of RSS is Low & Then Low EW \\
\hline 3 & When value of RSS is Medium & Then Medium EW \\
\hline 4 & When value of RSS is High & Then High EW \\
\hline 5 & When value of RSS is Very High & Then Very High EW \\
\hline
\end{tabular}

By focusing on $e q^{n}(18)$ the redundant rules given in Table 1 are replaced by new final rules given by Table 4 .

Parameter Settings

\begin{tabular}{ll}
\hline Algorithm & Parameters \\
\hline PSO & $N P=100 ; D=3 ; G_{\max }=200 ; c_{1}, c_{2}, c_{3}=1.494 ; w=0.729, \mathrm{DOI}=0.01$ \\
HPSO & $N P=100 ; D=3 ; G_{\max }=200 ; c_{1}, c_{2}, c_{3}=1.494 ; \eta=0.1 ; w=0.729, \mathrm{DOI}=0.01$ \\
BBO & $N P=100 ; D=3 ; G_{\max }=200 ; p_{m}=0.05, \mathrm{DOI}=0.01$ \\
FA & $N P=100 ; D=3 ; G_{\max }=200 ; \alpha=0.2 ; \gamma=0.96, \mathrm{DOI}=0.01$ \\
GWO & $N P=100 ; D=3 ; G_{\max }=200 ; \mathrm{a}=[2$ to 0$] ; \mathrm{C}=[0$ to 2$], \mathrm{DOI}=0.01$ \\
GWO-FA & $N P=100 ; D=3 ; G_{\max }=200 ; \mathrm{a}=[2$ to 0$] ; \mathrm{C}=[0$ to 2$], \alpha=0.2 ; \gamma=0.96$, \\
& $\mathrm{DOI}=0.01$ \\
\hline
\end{tabular}

\section{Simulation Results and Performance Analysis}

The proposed GWO-FA algorithm with fuzzy is implemented in MATLAB environment with a single anchor node. A cubical area of $10 l \times 10 l \times 10 l$ is assumed where an anchor node is kept fixed and target nodes mobile. After a fixed interval, an anchor node positioned at the top layer having transmission range $R=10 \mathrm{l}$. With the movement of target node, an anchor node can communicate only if they are within the range of anchor node. As soon as target nodes drop within the anchor node's range, six virtual anchors are projected using parasol projection and out of these six three virtual anchor nodes are selected to determine 3D location. The edge weights between every target node and an anchor, virtual anchors is used and modelled using Fuzzy Logic System (FLS). After modelling them using FLS the optimization of these edge weights is done using various meta-heuristics. The RSS parameter is considered as the simulation parameter and is given as

$$
R S S=\text { Sending Power }-(\text { DOI adjusted path loss }+ \text { Noise })
$$

DOI Adjusted Path loss $=$ Path Loss $\times K_{i}$; Where $K_{i}$ is used to represent variations in path loss in different directions.

In the initial step, we have assumed anchor at the centre position of the top layer and afterwards it moves randomly at regular intervals of time. We have assumed nine movements of an anchor node in this paper and seen a better performance in terms of location identification of target nodes with better accuracy.

Thirty trials with each meta-heuristics are applied to optimize RSS value and the edge weights, Fig ---- and ---- shows that the results obtained with proposed GWO-FA algorithm provides better performance as compared with other meta-heuristics.

The below mentioned parameters are assumed for comparison of proposed scheme with other meta-heuristics.

1. Linear Combination: 


$$
L C=\frac{a \times \text { promixity }+b \times R S S}{a+b}
$$

Where

$$
\text { promixity }=\frac{1}{\text { distance between anchor node and target node }}
$$

Where, $\mathrm{a}$ and $\mathrm{b}$ values lies between 0 and 1 .

2. Location Error: $L E=\sqrt{\left(x_{e s t}-x_{a c t}\right)^{2}+\left(y_{e s t}-y_{a c t}\right)^{2}+\left(z_{e s t}-z_{a c t}\right)^{2}}$

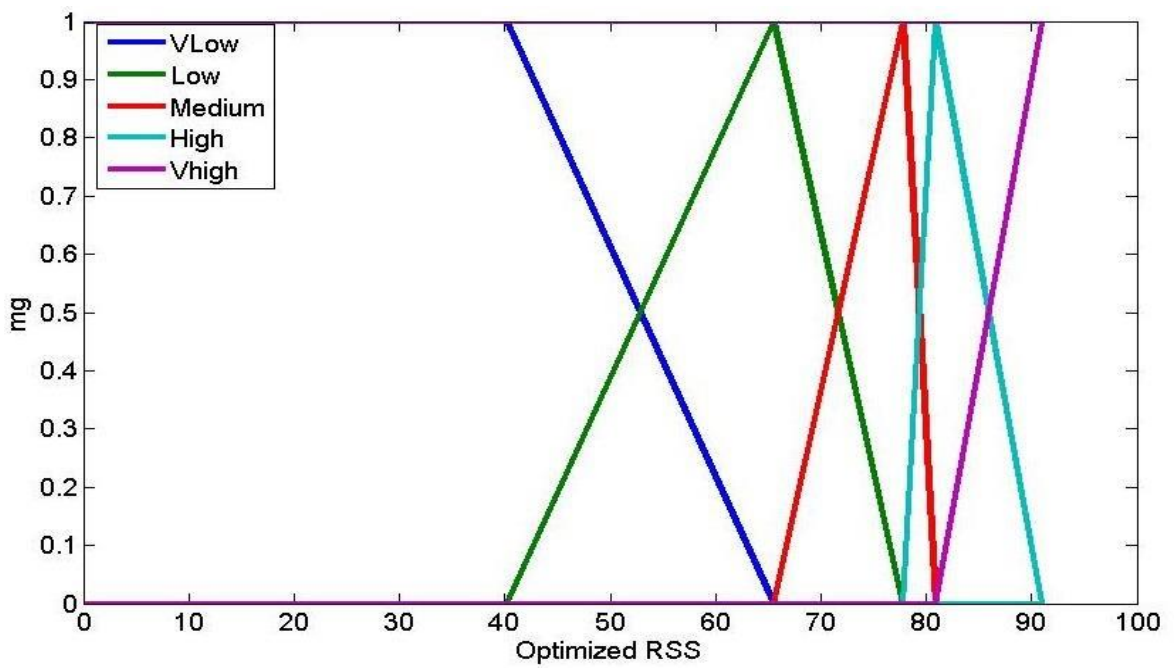

Figure 5: Optimized RSS fuzzy membership functions using GWO-FA

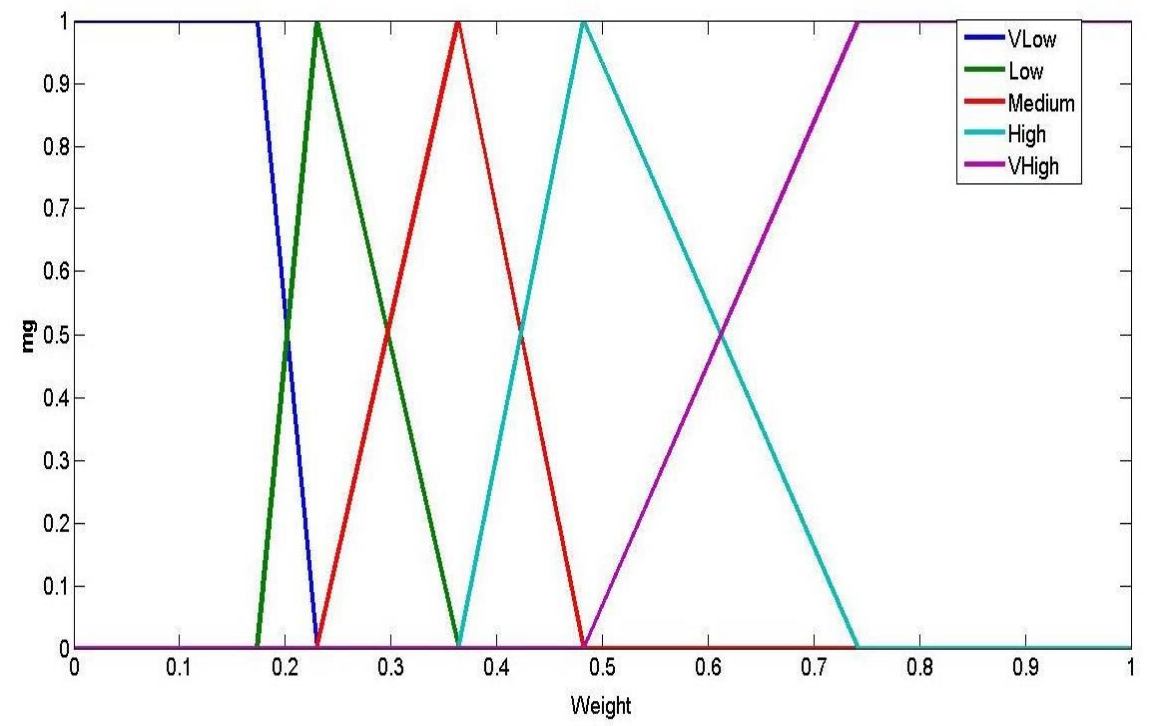

Figure 6: Optimized edge weights fuzzy membership functions using GWO-FA

3. Average Location Error:

$$
A L E=\frac{\sqrt{\left(x_{e s t}-x_{a c t}\right)^{2}+\left(y_{\text {est }}-y_{\text {act }}\right)^{2}+\left(z_{\text {est }}-z_{\text {act }}\right)^{2}}}{\text { number of target nodes }}
$$


Where $\left(x_{\text {est }}, x_{a c t}, y_{\text {est }}, y_{a c t}, z_{\text {est }}, z_{a c t}\right)$ are actual and the computed coordinates of a target node.

In this paper, the proposed algorithm GWO-FA is compared with other meta-heuristics available in literature. The simulation results of proposed scheme provide better results using a single anchor node with less localization error.

\begin{tabular}{|c|c|c|c|c|}
\hline $\begin{array}{l}\text { Static/ } \\
\text { Mobile }\end{array}$ & Technique & Max error & Min error & Average Error \\
\hline Static & Centroid & 3.892 & 0.129 & 2.910 \\
\hline Static & $\begin{array}{l}\text { Weighted } \\
\text { Centroid }\end{array}$ & 2.751 & 0.0610 & 1.432 \\
\hline Static & HPSO+Fuzzy & 0.991 & 0.088 & 0.812 \\
\hline Static & BBO+Fuzzy & 0.868 & 0.079 & 0.658 \\
\hline Static & BFO+Fuzzy & 0.831 & 0.067 & 0.551 \\
\hline Static & IWO+Fuzzy & 0.796 & 0.043 & 0.486 \\
\hline FStatic & TLBO+Fuzzy & 0.768 & 0.032 & 0.481 \\
\hline Static & MIWO+Fuzzy & 0.594 & 0.025 & 0.475 \\
\hline Static & GWO-FA+Fuzzy & 0.531 & 0.015 & 0.452 \\
\hline Mobile & HPSO+Fuzzy & 0.681 & 0.078 & 0.449 \\
\hline Mobile & BBO+Fuzzy & 0.768 & 0.059 & 0.512 \\
\hline Mobile & $\mathrm{BFO}+\mathrm{Fuzzy}$ & 0.712 & 0.057 & 0.431 \\
\hline Mobile & IWO+Fuzzy & 0.796 & 0.043 & 0.486 \\
\hline Mobile & TLBO+Fuzzy & 0.723 & 0.002 & 0.429 \\
\hline Mobile & MIWO+Fuzzy & 0.594 & 0.005 & 0.288 \\
\hline Mobile & GWO-FA+Fuzzy & 0.531 & 0.015 & 0.227 \\
\hline
\end{tabular}




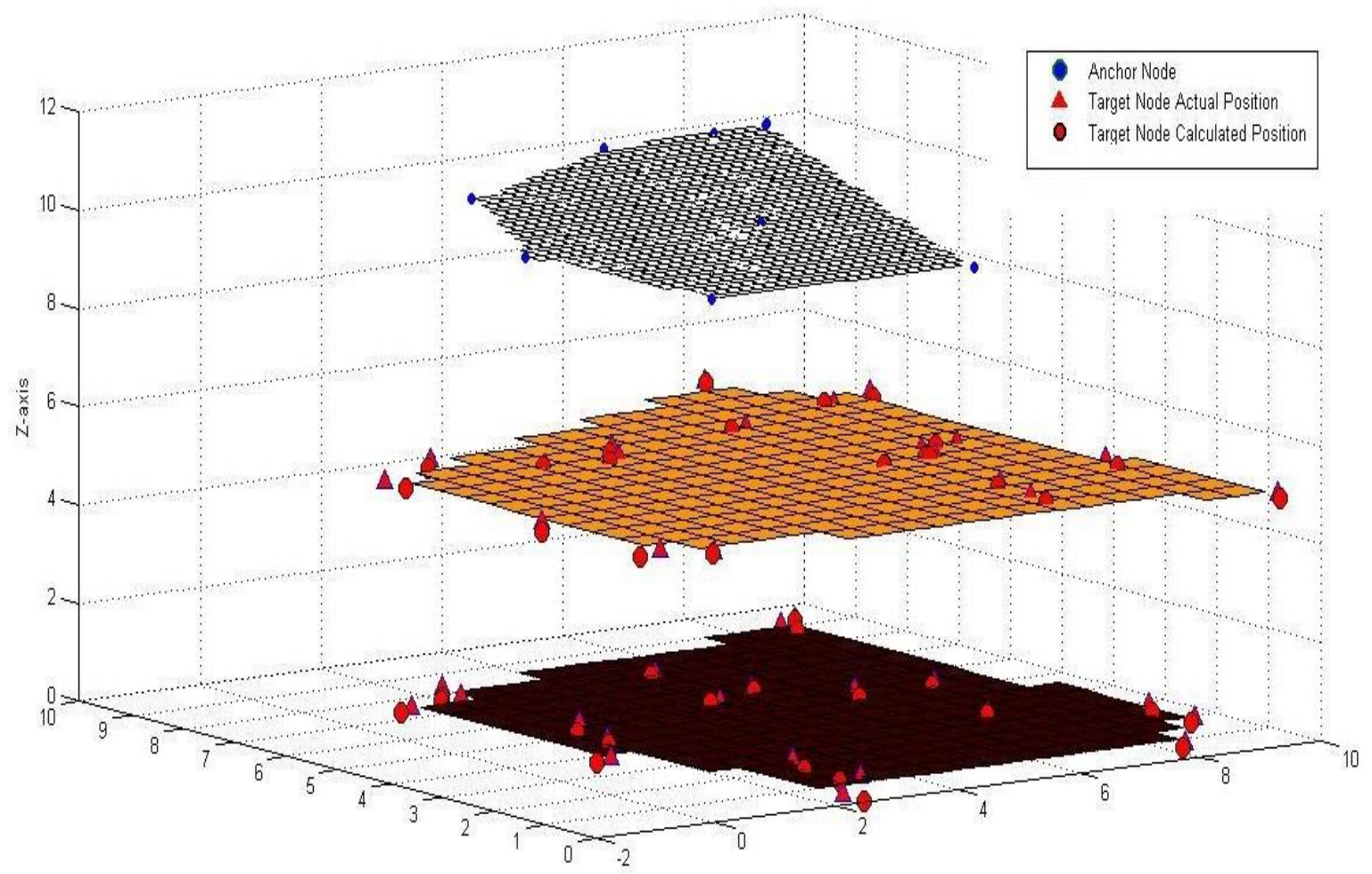

Y-axis

$x$-axis

Figure 7: GWO-FA based node localization
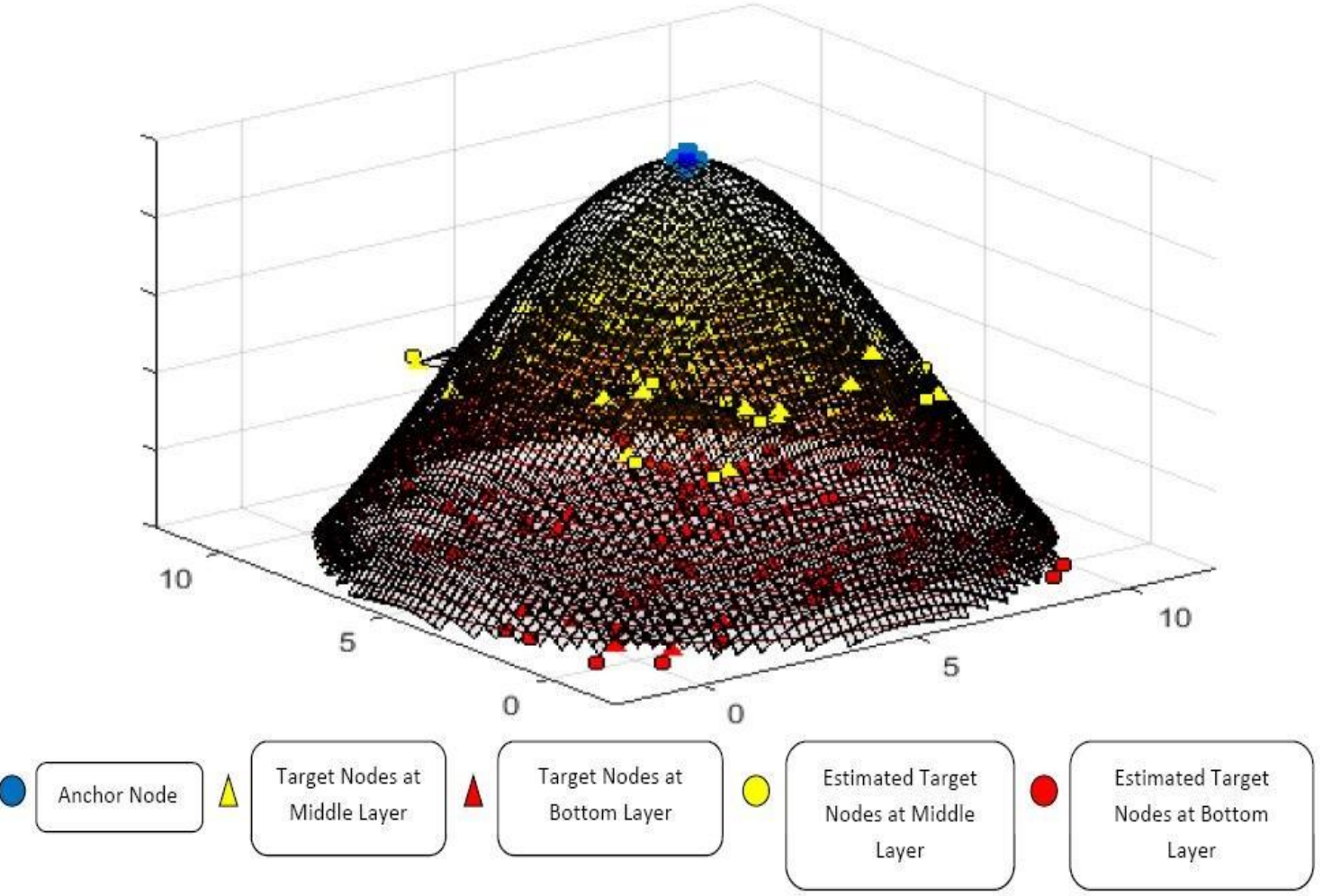

Figure 8: RF GWO-FA based node localization 


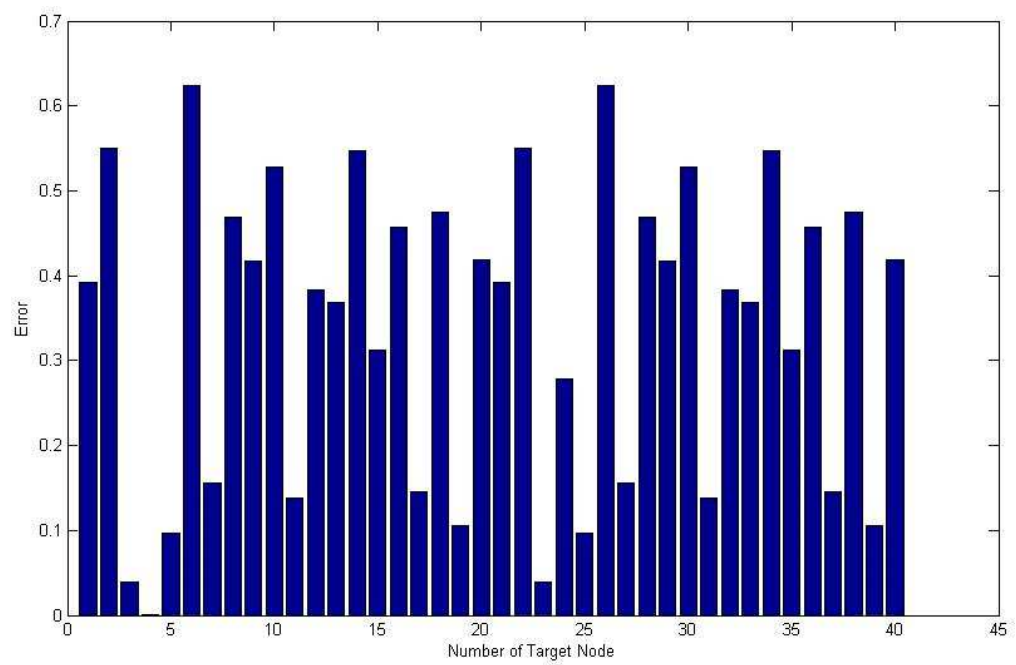

Figure 9: RF GWO-FA based node distance estimation between actual and estimated nodes

\section{Conclusions and Future Scope}

A method for range-free 3D node localization has been presented using a variety of metaheuristics methods. The top layer is considered to have a single mobile anchor node, and the target nodes and anchor node are deployed across three layer boundaries in an anisotropic environment. The anchor node traverses the top layer, whereas the target nodes are spread throughout the lower levels. To model the change in environmental conditions, a superimposed fading model is utilised. To establish the 3D coordinates of each target node, an unique notion of virtual anchor nodes is proposed. The non-linear relationship between Received Signal Strength (RSS) and distance has been minimised by assigning edge weights to each target node and anchor node in order to identify the target node's position. To reduce computational complexity, we modelled RSS and edge weights using a Fuzzy Logic System (FLS). Additionally, the RSS and edge weights membership function bases are optimised using HPSO, BBO, and BOA to decrease position error. The proposed algorithm's simulation results were compared to those of previously published static methods such as centroid, weighted centroid, RFHPSO, RFBBO, RFBFO, RFIWO, and RFBOA, and it was determined that the proposed method, RFBOA, achieves a higher level of localization accuracy than the others. In the future, hybrid localization, which combines range-based and range-free techniques with the application of artificial intelligence, may be considered for improved localization accuracy.

\section{References}

[1] X.-S. Yang, Firefly algorithm, stochastic test functions and design optimisation, arXiv preprint arXiv:1003.1409.

[2] T. Arampatzis, J. Lygeros, S. Manesis, A survey of applications of wireless sensors and wireless sensor networks, in: Proceedings of the 2005 IEEE International Symposium on, Mediterrean Conference on Control and Automation Intelligent Control, 2005., IEEE, 2005, pp. 719-724.

[3] Z. Rongbai, C. Guohua, Research on major hazard installations monitoring system based on wsn, in: 2nd International Conference on Future Computer and Communication, Vol. 1, IEEE, 2010, pp. V1-741.

[4] L. Ghelardoni, A. Ghio, D. Anguita, Smart underwater wireless sensor networks, in: IEEE 27th Convention of Electrical and Electronics Engineers in Israel, IEEE, 2012, pp. 1-5. 
[5] Y. Singh, S. Saha, U. Chugh, C. Gupta, Distributed event detection in wireless sensor networks for forest fires, in: UKSim 15th International Conference on Computer Modelling and Simulation, IEEE, 2013, pp. 634-639.

[6] N. Patwari, J. N. Ash, S. Kyperountas, A. O. Hero, R. L. Moses, N. S. Correal, Locating the nodes: cooperative localization in wireless sensor networks, IEEE Signal processing magazine 22 (4) (2005) 54-69.

[7] N. Bulusu, J. Heidemann, D. Estrin, “"GPS-Less Low-Cost Outdoor Localization for Very Small Devices", IEEE Personal Communications 7 (5) (2000) 28-34.

[8] G. Gao, L. Lei, "An Improved Node Localization Algorithm Based on DV-HOP in WSN", in: 2nd International Conference on Advanced Computer Control (ICACC), Vol. 4, IEEE, 2010, pp. 321-324.

[9] L. Doherty, et al., "Convex Position Estimation in Wireless Sensor Networks", in: Proceeding of INFOCOM, Vol. 3, 2001, pp. 1655-1663.

[10] Y. Zhou, X. Ao, S. Xia, "An Iimproved APIT Node Self-Localization Algorithm in WSN", in: 7th World Congress on Intelligent Control and Automation (WCICA 2008), 2008, pp. 7582-7586.

[11] J. Bachrach, C. Taylor, "Localization in Sensor Networks", Handbook of Sensor Networks (2005) 277-310.

[12] R. Teng, B. Zhang, "On-Demand Information Retrieval in Sensor Networks With Localised Query and Energy-Balanced Data Collection”, Sensors 11 (1) (2010) 341-361.

[13] B. Zhang, J. Fan, G. Dai, T. H. Luan, A hybrid localization approach in 3d wireless sensor network, International Journal of Distributed Sensor Networks 11 (10) (2015) 692345.

[14] S. Yun, J. Lee, W. Chung, E. Kim, S. Kim, A soft computing approach to localization in wireless sensor networks, Expert Systems with Applications 36 (4) (2009) 7552-7561.

[15] H. Xiong, M. L. Sichitiu, A lightweight localization solution for small, low resources wsns, Journal of Sensor and Actuator Networks 8 (2) (2019) 26.

[16] S. Sivasakthiselvan, V. Nagarajan, A new localization technique for node positioning in wireless sensor networks, Cluster Computing 22 (2) (2019) 4027-4034.

[17] H. Chen, G. Tan, Adaptive iteration localization algorithm based on rssi in wireless sensor networks, Cluster Computing 22 (2) (2019) 3059-3067.

[18] T. LN Nguyen, T. D Vy, Y. Shin, An efficient hybrid rss-aoa localization for 3d wireless sensor networks, Sensors 19 (9) (2019) 2121.

[19] A. Kumar, A. Khosla, J. S. Saini, S. S. Sidhu, Range-free 3d node localization in anisotropic wireless sensor networks, Applied Soft Computing 34 (2015) 438-448.

[20] G. Sharma, A. Kumar, Fuzzy logic based 3d localization in wireless sensor networks using invasive weed and bacterial foraging optimization, Telecommunication Systems 67 (2) (2018) $149-162$.

[21] S. Lee, C. Park, M. J. Lee, S. Kim, Multihop range-free localization with approximate shortest path in anisotropic wireless sensor networks, EURASIP Journal on Wireless Communications and Networking 2014 (1) (2014) 80.

[22] Y.-S. Chen, Y.-J. Ting, C.-H. Ke, N. Chilamkruti, J. H. Park, Efficient localization scheme with ring overlapping by utilizing mobile anchors in wireless sensor networks, ACM Transactions on Embedded Computing Systems (TECS) 12 (2) (2013) 1-18.

[23] V. K. Chaurasiya, N. Jain, G. C. Nandi, A novel distance estimation approach for 3d localization in wireless sensor network using multidimensional scaling, Information Fusion 15 (2014) 5-18.

[24] S. Mirjalili, S.M., Lewis, A. (2014). Grey wolf optimizer, Advances in Engineering Software, 69, pp 46-61

[25] X. S Yang: 'Firefly algorithm, levy flights and global optimization', Research and Development in Intelligent Systems, 2010, pp. 209-218 
[26] Janez, B., Mirjam, M., Borko, S. B.: 'The 100-Digit Challenge: Algorithm', 2019, pp. 1926. 